\title{
Simulated Race for Exam Grades
}

\author{
Drago Matko*, Ales Belič, S. Blažič \\ Faculty of Electrical Engineering, University of Ljubljana, Trzaska 25, 1000 Ljubljana, Slovenia. \\ *drago.matko@fe.uni-lj.si
}

\begin{abstract}
The idea on how to motivate students to learn subjects such as Comput er Aided Control Systems Design (CACSD) at the Faculty of Electrical Engineering, University of Ljubljana is presented. A simulated race instead of exam is tested, meaning that the students are graded according to the success achieved in the competition. The aim of the competition is set by the professor, and the students have to design a con troller to achieve the bes $t$ possible result they can. The $p$ aper concludes with the descr iption of solutions applied by s tudents and the $f$ aculty experiences with the course.
\end{abstract}

\section{Introduction}

The standard approach to teaching/examining Computer Aided Control Systems Design is to give lessons on control algorithms and design tools, to give students exercises to be completed in a classroom equipped with computers, to provide similar exercises in the written part of the exam, and to complete the exam with oral questions/answers.

Modern education demands a significantly different approach to classical methods. Students must get new perspectives and learn how to solve practical problems. The mere transmission of facts to them must be replaced by giving them knowledge of how to cope with the challenges of the market economy.

In the paper a new idea in examining Computer Aided Control Systems Design (CACSD) - namely a competition - is presented. It is organized as follows: In Section 2 the idea of the virtual race is presented. The subjects of the virtual race, used in the last four years at the Faculty of Electrical Engineering, University of Ljubljana, are given in Section 3. In Section 4, the solutions, applied by students, and our experience with the virtual race are given, while Section 5 presents the students' view of the examination.

\section{The Idea of the Examination by Virtual Race}

The main idea for the exam is to be a competition or a race. The student's mark depends on his/her position in the race. The student winning the competition gets the best mark. The distribution of marks equals the distribution of average student marks in the classroom. This measure should prevent the copying of solutions among students. The object of the competition is different every year, thereby preventing copying of the solutions from previous years.

The competition is performed by simulation using Simulink/Matlab. The plant (its structure) is known to the students; however, some parameters remain unknown (only lower and upper bounds are known). The inputs and outputs of the plant are defined as an interface to the controller to be designed by students as a single block. The students are totally free in designing the controller; they should use their knowledge to obtain the best result.

The problems to be optimally solved by students are not classical in nature. For example, the printed information is not always the transfer function and the criterion function. Instead, problems are structured to encourage curiosity, emphasize mathematical describeability, be numerically non-demanding and include some complexity.

Mathematical describability is necessary since the problem is only given to the students in the form of equations, not as a simulation model. In our approach, students have to build a model according to the given equations and to design a controller which controls the simulated model optimally according to the specified criterion. Also, the interface between the controller and process is exactly specified.

All the students (regardless of their computers' power) must have equal possibilities; therefore, it is important how numerically (non)demanding a problem is. The complexity of the model enables students to show their creativity. 


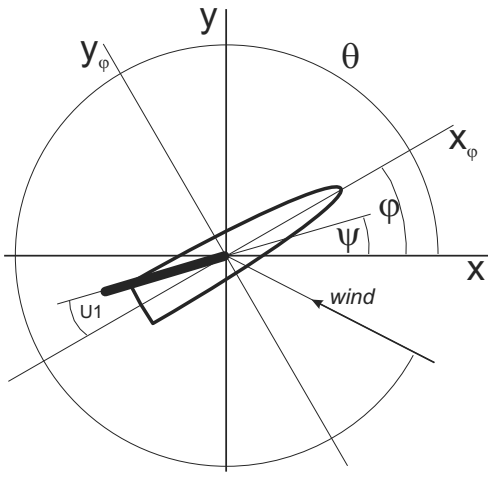

Figure 1. The top view of the model of the surf

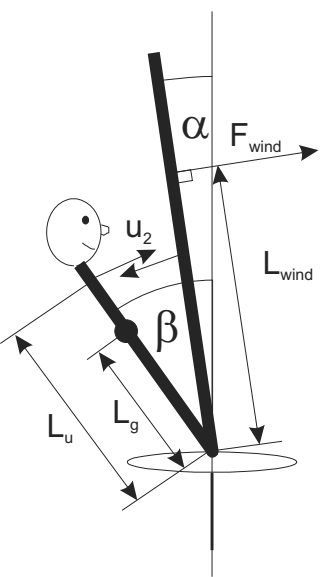

Figure 2. The front view of the model of the surf.

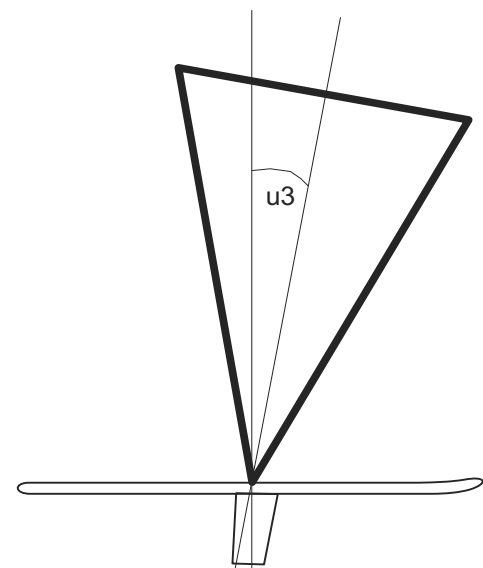

Figure 3. The side view of the model of the surf.
The students have three to five weeks to design the controller. During this time, they can come to the professor for discussions; they can also test their controller on the test model.

Even though students are highly motivated to obtain good marks, there are a few individuals in every classroom who try to minimize their effort and copy the solution. There is an effort to suppress this anomaly in the competition; better results get better marks. In addition, a traditional oral exam concerning the subject of the applied controller, as well as penalties for equal solutions, should prevent copying.

This new approach also tries to break the traditional notion that students are allies against the professor and introduce a new notion with the professor and student being allies and with students competing against each other. This practice was introduced four years ago; and, since every year the practical problem to be solved is different, four problems being solved by students are given in the next section.

\section{Subjects of the Virtual Race}

Four different competition subjects have so far been used. They are the inverted pendulum, the solar sail race, the giant slalom contest, and the surf regatta. All four objects are non-linear processes. Besides this, the control variable or variables respectively were also saturated. In all four cases, minimal time was the criterion function.

In the first year, the goal was to transfer an inverted pendulum from one point (standing upright) to another point 4.8 meters away and to remain there in an upright position (within $\pm 2.5^{\circ}$ tolerance) for five seconds. In the second year, the goal was to transfer a solar sail from a circular $700 \mathrm{~km}$ orbit to a geostationary orbit (only to cross it, not to remain in it) as quickly as possible. In the third year, a giant slalom contest was performed. The model of the skier was completely known. However, the course setting was unknown to students; it was given to them five minutes before the start. In these five minutes they could make final adjustments to the controller. The contest consisted of two runs: a simple run and a more sophisticated run. For a positive mark, only the first run had to be completed without any fault (missing gates).

In the fourth year the subject of the competition was a surf regatta. The model of the surf was completely known to the students and so was the course: to encircle in the prescribed direction the two buoys and to return to the starting point (the finish line was \pm 50 meters apart from the starting point). Unknown (in advance) were the wind direction and force. The model of the surf is illustrated in Figures 1 to 3. The surf is driven by the wind according to

$$
F_{\text {wind }}=p_{\text {wind }} S\left(v_{w}-v_{\operatorname{surf}_{\Theta}}\right) \cos \alpha \sin (\Theta-\psi)
$$

where $p_{\text {wind }}$ is the wind pressure, $S$ is the area of the surf sail, $v_{w}$ the velocity of the wind, $v_{\text {surf }_{\Theta}}$ the velocity of the surf in the direction of the wind, $\Theta$ the wind direction angle, $\psi$ the angle of the sail according to the fixed co-ordinate system, and $\alpha$ the lateral angle of the sail (limited to $\pm \pi / 2$ ) (see Figure 2).

The wind force $F_{\text {wind }}$ is decomposed into two forces, into the force $F_{\text {wind }}$ pushing the surf forward and into the force $F_{\text {wind }_{90}}$ pushing the surf perpendicular to the direction of the surf 


$$
\begin{gathered}
F_{\text {wind }_{\varphi}}=F_{\text {wind }} \sin (\phi-\varphi) \cos \alpha \\
F_{\text {wind }_{90}}=-F_{\text {wind }} \cos (\psi-\varphi) \cos \alpha
\end{gathered}
$$

where $\varphi$ is the surf board direction angle. Due to these two forces, the surf is moving into the direction of the surf board $\left(x_{\varphi}\right)$ and perpendicular to it $\left(y_{\varphi}\right)$.

$$
\begin{aligned}
& m \ddot{x}_{\varphi}=F_{\text {wind }_{\varphi}}-k_{2} \dot{x}_{\varphi} \\
& m \ddot{y}_{\varphi}=F_{\text {wind }_{90}}-k_{3} \dot{y}_{\varphi}
\end{aligned}
$$

where $m$ is the mass of the surf (surfer and board) and $k_{2}, k_{3}\left(k_{2} \ll k_{3}\right.$ due to the keel) the resistance constants into directions $x_{\varphi}$ and $y_{\varphi}$, respectively.

The velocity of the surf into the direction of the wind is given by

$$
v_{\text {surf }_{\Theta}}=\dot{x}_{\varphi} \cos (\Theta-\varphi)-\dot{y}_{\varphi} \sin (\Theta-\varphi)
$$

The movement of the surf in the fixed coordinate system is obtained by the following transformations:

$$
\begin{aligned}
& \dot{x}=\dot{x}_{\varphi} \cos \varphi-\dot{y}_{\varphi} \sin \varphi \\
& \dot{y}=\dot{x}_{\varphi} \sin \varphi+\dot{y}_{\varphi} \cos \varphi
\end{aligned}
$$

The lateral dynamics of the surf (sail and surfer) is the dynamics of two coupled inverted pendulums (see middle part of Figure 1).

$$
\begin{gathered}
I_{\text {sail }} \ddot{\alpha}=-F_{\text {wind }} L_{\text {wind }}+u_{2} L_{u} \\
I_{\text {surfer }} \ddot{\beta}=-u_{2} L_{u}+m_{\text {surfer }} g \sin \beta L_{g}
\end{gathered}
$$

where $I_{\text {sail }}$ and $I_{\text {surfer }}$ are the momenta of inertia of the sail and surfer respectively (around the horizontal axis), $u_{2}$ the force of the surfer to the sail (limited to $\pm 800 \mathrm{~N}), L_{u}, L_{\text {wind }}$ and $L_{g}$, the levers of the surfer's force to the sail, wind and gravity respectively, $m_{\text {surfer }}$ the mass of the surfer, $g$ the gravity constant, and $\beta$ the lateral angle of the surfer (limited to $\pm \pi / 2$ ). The gravity force of the sail was neglected.

The angle of the sail relative to the wind is determined by

$$
\psi=-u_{1}+\varphi
$$

where $u_{1}$ is the angle of the sail relative to the surf board.

The turning of the surf is obtained by placing the wind force center point forwards and backwards, as illustrated in the right part of Figure 1. The angle of the surf board is determined by

$$
I_{\text {surf }_{v}} \ddot{\varphi}=-k_{4} \dot{\varphi}+F_{\text {wind }} L_{\text {wind }} \sin u_{3}
$$

where $I_{\text {surf }}$ is the momentum of inertia of the surf board

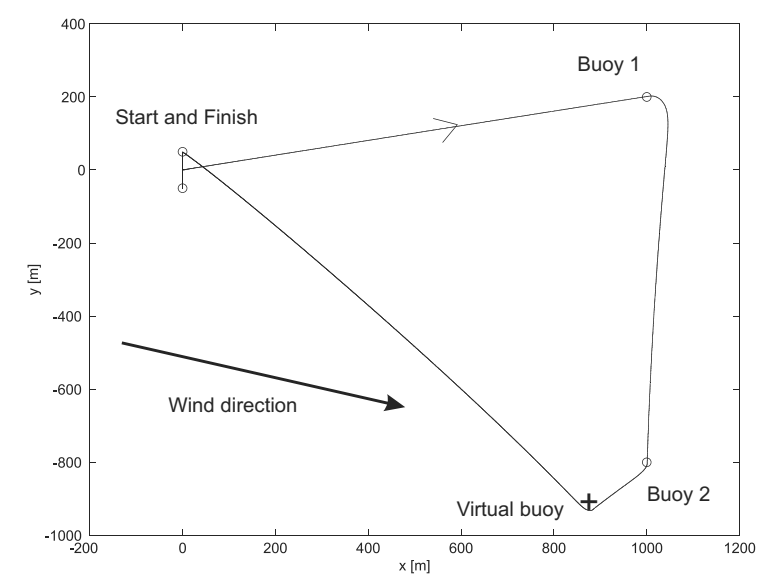

Figure 4. The course of the surf regatta.
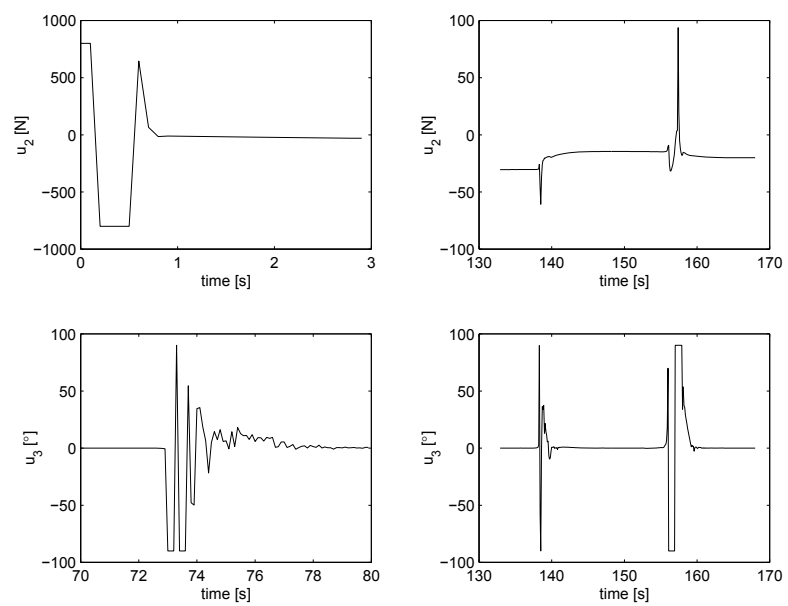

Figure 5. The time history of the control variables: the angle of the sail relative to the surf (uppermost), two details of the force stabilizing the inverted pendulum system surfer - sail (middle), two details of the angle of the mast in the direction forward - backward (lower).

and surfer around the vertical axis, $k_{4}$ the coefficient of resistance, and $u_{3}$ the angle of the mast in the direction forwards-backwards. The interface between the controlled object (the surfer) and the controller to be designed by students was specified.

\section{Solutions and Experiences}

According to our observations the great majority of students is very enthusiastic about the competition. The problems and results are discussed among students, but the details and new ideas are not revealed. In the case of the inverted pendulum, solar sail, and giant slalom contest, the most favored approach applied by students was to linearize the models in different operating points, to optimize the controller parameters by optimization pro- 

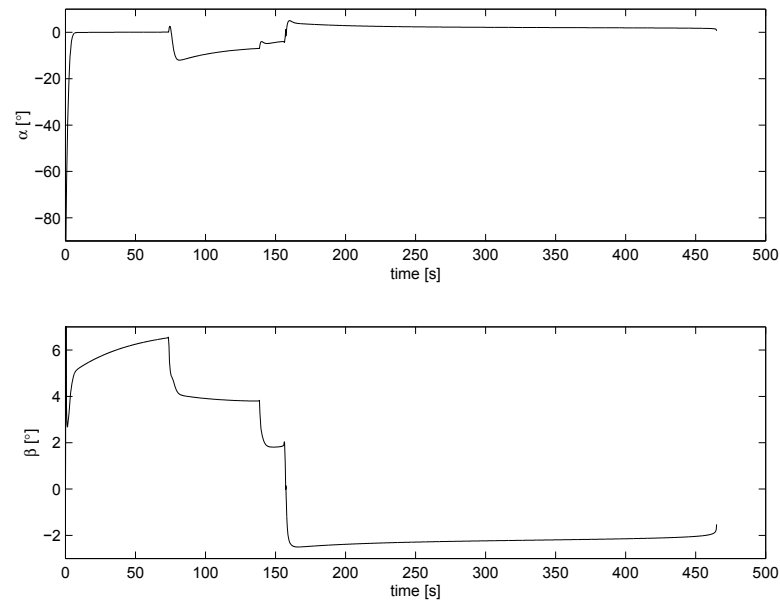

Figure 6. The time course of the lateral angles of the sail and surfer.

cedures, and to schedule the controllers according to the state of the process. In the case of the surf regatta, the tuning of controllers was not very important in comparison to the optimal course setting. This fact was realized by the students quite early; and, in the shortage of time, the concentration was focused on the course setting.

Figures 4, 5, and 6 illustrate the results of a typical solution. In Figure 4, depicting the course, it can be seen that the used course was detoured to sail quicker against the wind. With respect to the control strategy a detour may be obtained by setting an additional (virtual) buoy. In Figure 5 the time history of control variables is shown. In the uppermost part of the figure the angle of the sail relative to the surf direction is depicted. In the middle part of the figure two details of the force stabilizing the inverted pendulum system surfer-sail are shown. The beginning of the race, i.e., lifting the sail from the horizontal position is depicted on the left, while the segment involving the turn around the second buoy and the virtual buoy set to make a detour is shown in the right-hand side of the middle part of the figure. The lower part of the figure contains two details of the third control variable - the angle of the mast in the direction forward and backward. In the left part the time history during the turning around the first buoy and in the right part the turning around the second and the virtual buoys are shown, respectively. It can be seen that the fixed controller was tuned for the turning around the second and the virtual buoys; the control signal during the turning around the first buoy is quite oscillatory.

In Figure 6 the time history of the lateral angles of the sail and surfer are presented. The optimal position of the sail is vertical. However, if the wind is too strong to

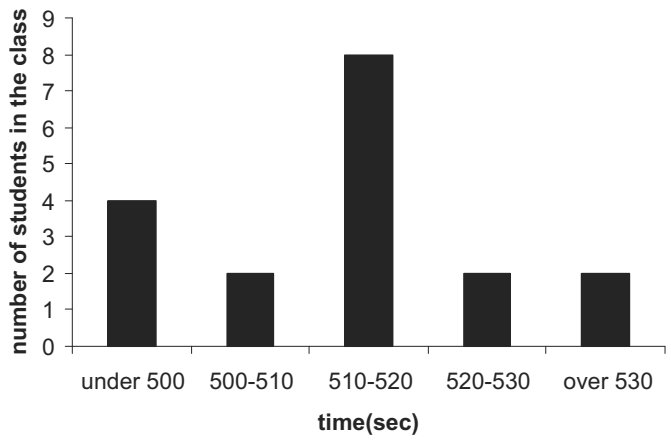

Figure 7. The distribution of scores for the surf regatta.

be compensated by the gravity force of the surfer, the sail must be inclined in order to reduce the wind force. In the actual race the wind was not so strong, so the reference value for the upper part of the figure is zero. It can be seen again that the controller was tuned for the last part of the race; it was too "lazy" in the segment of the race prior to the first buoy.

The distribution of scores for the inverted pendulum and solar sail competitions had a normal (Gaussian) character. The distribution for the giant slalom competition had an "inverted" normal distribution. The reasons for this seem to be the non-selective course setting and too much time ( 5 weeks) elapsing between giving the exercise to the students and the actual competition. Also, the distribution of the surf regatta, shown in Figure 7, does not exhibit Gaussian shape. The reason for this unexpected phenomenon is that the optimal course on the part of the path which was in the direction against the wind was not to go directly to the next buoy but to make a detour. Students using this detoured course gained better results, but not all students realized this strategy. The consequence is a double peak distribution for students' scores.

\section{The Students' Perspective}

The non-standard examination approach was wellaccepted by students, and the theory taught in the standard course was recognized only as a necessary but not sufficient tool in solving practical problems. The key point of a good score was innovation. This and new, fresh ideas is what enterprises, competing on the market, expect from an engineer. Innovative thinking cannot 
be taught, but modern education should at least stimulate and remunerate this approach by setting priority in scoring to ideas rather than to the ability of memorizing. Another very important point is motivation. According to the assertions of students, even though the amount of time spent on the Virtual race project was greater than the amount of time spent on other projects, students enjoyed studying the course and did not regret have chosen it. A learning-by-game approach seems to be more attractive.

The attractiveness of the race subject seems to be an important point. Regarding this issue the surf regatta was the most exciting. Some of students even started wind-surfing after the course.

\section{Conclusions}

A new practice in examining the subject of CACSD has been introduced in the last four years. A virtual race - a competition between students - enables them to demonstrate their creativity. Four objects of the competition are given in the paper: the inverted pendulum, the solar sail race, the giant slalom contest, and the surf regatta. The description of the most favourable controller design solutions, the experiences with the virtual race, and the distributions of results are also given. It is the experience of the authors that students accepted the competition as a form of the written part of exams with enthusiasm. Accordingly, this practice will be continued in the future.

\section{References}

[1] Matlab Users Guide, Mathworks Inc., Natick, 1992.

[2] Simulink Users Guide, Mathworks Inc., Natick, 1992.

[3] S. Eguchi, N. Ishii, H. Matsuo, Fundamental Studies of Designing a Solar Sail Orbit, In Proc. 17th International Symposium on Space Technology and Science, (Tokyo, Japan, 1990), 1990.

[4] C. Jack, C.S. Welch, Solar Kites: Small Solar Sails with no Moving Parts, In Proc. 47th International Astronautical Congress (Beijing, China, 1996), 1996. 